Research Article

\title{
Effective Extraction of Palmatine and Berberine from Coptis chinensis by Deep Eutectic Solvents-Based Ultrasound- Assisted Extraction
}

\author{
Lijing Li $\mathbb{D}^{1},{ }^{1}$ Dong Zhang $\mathbb{D}^{1},{ }^{1}$ Yuejie Wang $\mathbb{D},{ }^{1}$ Fangxin Liu $\mathbb{D}^{1},{ }^{1}$ Yang Xu $\mathbb{D},{ }^{2}$ \\ and Huiwei Bao $\mathbb{D}^{1}$ \\ ${ }^{1}$ College of Pharmacy, Changchun University of Chinese Medicine, Changchun 130117, China \\ ${ }^{2}$ College of Pharmacy, Baicheng Medical College, Baicheng 137000, China \\ Correspondence should be addressed to Yang Xu; 251748191@qq.com and Huiwei Bao; baohuiwei@163.com
}

Received 11 March 2021; Revised 24 July 2021; Accepted 6 August 2021; Published 13 August 2021

Academic Editor: Marianela Savio

Copyright (C) 2021 Lijing Li et al. This is an open access article distributed under the Creative Commons Attribution License, which permits unrestricted use, distribution, and reproduction in any medium, provided the original work is properly cited.

\begin{abstract}
The effective components of Coptis chinensis were extracted by ultrasound-assisted technology, and the contents of palmatine and berberine were used as indexes by using Coptis chinensis as raw material and eutectic solvent as extractant. In addition, the effects of hydrogen bond donor type, molar ratio of hydrogen bond donor to acceptor, material-liquid ratio, water content of eutectic solvent system, sonication time, power, and ultrasonic temperature on the extraction rate of palmatine and berberine were studied. The optimum extraction technology of palmatine and berberine from Coptis chinensis was determined by single-factor experiment and response surface optimization test. As a result, it showed that the eutectic solvent system was constructed with choline chloride as hydrogen bond acceptor and phenol as hydrogen bond donor, with a molar ratio of $1: 3$. In addition, water content of the eutectic solvent system was $30 \%$, ratio of material to liquid was $30 \mathrm{~g} / \mathrm{mL}$, ultrasonic time was $30 \mathrm{~min}$, ultrasonic power was $200 \mathrm{~W}$, and ultrasonic temperature was $60^{\circ} \mathrm{C}$. At this time, the contents of palmatine and berberine in Coptis chinensis were $16.7145 \mathrm{mg} / \mathrm{g}$ and $57.4013 \mathrm{mg} / \mathrm{g}$, respectively, which were predicted to be the same as the value, and the extraction effect was better than that of traditional extraction solvent method.
\end{abstract}

\section{Introduction}

As the dry rhizome of Coptis chinensis, C. deltoidea C. Y. Chenget Hsiao, and C. teeta Wall of Ranunculaceae, Coptis chinensis are commonly known as Weilian, Yalian, Yunlian [1], bitter in taste and cold-natured [2], which can rise or fall [3]. Besides, it belongs to the heart, liver, stomach, and large intestine meridians, with the effects of clearing heat, dryness, and dampness, purging fire, and detoxification $[1,4]$. In addition, Coptis chinensis was first published in the Classic of Shennong Materia Medica and was listed as a top-grade medicine [5]: "the smell is bitter, cold, and nontoxic." The chemical constituents of Coptis chinensis include alkaloids, lignans, flavonoids, and acidic components [6]. The main active components are alkaloids [7], of which berberine is the main component. In addition, the contents of alkaloids such as palmatine, jatrorrhizine, and magnoflorine are high [8].

As first reported by Abbott et al. in 2003 [9], deep eutectic solvents (DES) are a eutectic mixture composed of hydrogen-bonded acceptors and hydrogen-bonded donors of solid halide salts with a certain stoichiometric ratio [10]. It is usually composed of two or more components, and its melting point is significantly lower than that of each component [11]. It has the advantages of simple preparation, low cost, and no need for complex purification. In addition, it is also widely used in electrochemistry, preparation of nanomaterials, catalytic reaction, separation process, and preparation of functional materials, among others [12-15]. It is only necessary to mix the hydrogen bond donor and hydrogen bond acceptor at a certain molar ratio in the preparation of DESs. In addition, heat and stir at a certain 
temperature until a uniform liquid is formed [16]. As extractant, eutectic solvent is often combined with ultrasonic-assisted extraction [17], microwave-assisted extraction [18], hollow fiber extraction [19], and so on. Eutectic solvents mainly have the following advantages in extraction [20]: (1) the eutectic solvents synthesized by different components have different polarity, thus improving the extraction efficiency of different polarity analytes. (2) Eutectic solvents have the advantages of easily available raw materials, low toxicity, biodegradability, no pollution to the environment [21], easy regeneration, and so on, compared with the traditional methanol extractant.

In this study, we performed the isolation and extraction method of palmatine and berberine from Coptis chinensis based on the ultrasound-assisted eutectic solvent. Besides, the effects of different DESs and other extraction factors on the extraction of effective components of Coptis chinensis were investigated through single-factor experiment. In addition, we combined with response surface method to optimize the extraction process of effective components of Coptis chinensis and compared with traditional water extraction and alcohol extraction. This study is committed to providing a greener, efficient, economical, and environmentally friendly new method for the extraction of alkaloids from Coptis chinensis. It can better promote the in-depth development of Coptis chinensis and provide data support for the development of green chemistry and the research of natural products of traditional Chinese medicine.

\section{Experiment}

2.1. Instruments and Materials. Firstly, the chromatographic analysis was carried out by using a high-performance liquid chromatography system (HPLC-Huapu S3000 provided with a four-element low-pressure stirring pump, automatic injector, diode array detector model 1100, and chemical workstation). In addition, a JP-300G ultrasonic cleaner was purchased from Wuhan Jiapeng Electronics Co., Ltd. Moreover, QL-901 Vortex instrument was purchased from Haimen Qilinbell Instrument Manufacturing Co., Ltd. Besides, TGL-16 freezing centrifuge was purchased from Hunan Xiangyi Laboratory Instrument Development Co., Ltd.

In addition, the Coptis chinensis was produced in Sichuan Province, China, purchased from Jilin Pharmaceutical Store (Changchun); these samples meet the requirements of Coptis chinensis in Chinese Pharmacopoeia (2020 edition). Berberine hydrochloride $(\geq 85.9 \%)$ and palmatine hydrochloride $(\geq 85.7 \%)$ standards were used for HPLC determinations which were purchased from China Food and Drug Identification Institute. Chromatographic grade methanol and acetonitrile were purchased from Fisher Company of the United States. All reagents were of analytical grade. Phosphoric acid was purchased from Tianjin Guangfu Technology Development Co., Ltd., and ultrapure water was purchased from Hangzhou Wa Co., Ltd. Choline chloride was purchased from Shanghai Zhanyun Chemical Co., Ltd. Maltose, malic acid, lactic acid, fructose, xylitol, and citric acid were purchased from Zhengzhou Kangyuan
Chemical Products Co., Ltd. Phenol and acetic acid were purchased from Xilong Science Co., Ltd. Glycerin was purchased from Guangzhou Miya Cosmetics Co., Ltd. Propylene glycol was purchased from Tianjin Zhonghe Shengtai Chemical Co., Ltd. Urea was purchased from Tianjin Ruiji Chemical Co., Ltd.

2.2. HPLC Experimental Conditions. The chromatographic column was an Alltima ${ }^{\mathrm{TM}} \mathrm{C}_{18}$ column $(250 \mathrm{~mm} \times 4.6 \mathrm{~mm}$, $5 \mu \mathrm{m})$. Mobile phase included acetonitrile, $0.1 \%$, phosphoric acid aqueous solution (30:70, v:v). The selected wavelength was $345 \mathrm{~nm}$, the injection volume was $20 \mu \mathrm{L}$, the flow rate was $1.0 \mathrm{~mL} / \mathrm{min}$, and the column temperature was $30^{\circ} \mathrm{C}$.

2.3. Reference Solution. The reference substance of palmatine and berberine was precisely weighed and dissolved with methanol to obtain a mixed reference solution containing $0.572 \mathrm{mg} / \mathrm{mL}$ palmatine and $1.140 \mathrm{mg} / \mathrm{mL}$ berberine. The solution was precisely absorbed and diluted $1 \mathrm{~mL} 1: 10$ with methanol and filtered with a $0.22 \mu \mathrm{m}$ pore-size filter. In addition, the reference solution was obtained for further HPLC analysis.

2.4. Preparation of DESs. The hydrogen bond donor and hydrogen bond acceptor were mixed according to the molar ratio of Table 1 . Besides, it was heated in a water bath at $80 \sim 100^{\circ} \mathrm{C}$ and stirred well until a clear, transparent, and slightly viscous liquid solvent was obtained. For the selection of hydrogen bond receptors, choline chloride was selected, which was the mainstream and most widely used one in the market. In addition, for the selection of hydrogen bond donors, 11 kinds of maltose, malic acid, lactic acid, fructose, phenol, glycerol, propylene glycol, xylitol, urea, acetic acid, and citric acid were selected according to the principles of cost availability, safety, and controllability of raw materials.

\subsection{Palmatine and Berberine Extraction from Coptis chinensis} by DESs. $50 \mathrm{mg}$ Coptis chinensis was extracted (the powder passed through a 100-mesh sieve) and was added to eutectic solvent according to the ratio of material to liquid at $1: 30(\mathrm{~g} /$ $\mathrm{mL}$ ). Besides, it was placed in $60^{\circ} \mathrm{C}$ warm bath for $5 \mathrm{~min}$ and was vortex oscillated for $5 \mathrm{~min}$, and then ultrasonic extraction of $30 \mathrm{~min}(200 \mathrm{~W})$ at $60^{\circ} \mathrm{C}$ was conducted and it was vortex oscillated for $1 \mathrm{~min}$ and centrifugation of $3000 \mathrm{rpm} /$ min for $5 \mathrm{~min}$ was conducted.

2.6. Response Surface Test Design. Critical variables in the extraction process of Coptis chinensis were investigated, including DESs type, molar ratio, water content, materialliquid ratio, sonication time, ultrasonic temperature, and ultrasonic power. Several factors which had great influence on the extraction efficiency were selected as the independent variables of the response surface through the experimental design. The content of palmatine and berberine in Coptis chinensis was analyzed and optimized by response surface method. 
TABLE 1: Sources of deep eutectic solvents.

\begin{tabular}{lccc}
\hline Abbreviation & Hydrogen bond Receptor & Hydrogen bond Donor & Mole ratio \\
\hline DES-1 & Choline chloride & Maltose & $1: 1$ \\
DES-2 & Choline chloride & Malic acid & $1: 1$ \\
DES-3 & Choline chloride & Lactate & $1: 2$ \\
DES-4 & Choline chloride & Fructose & $1: 1$ \\
DES-5 & Choline chloride & Phenol & $1: 3$ \\
DES-6 & Choline chloride & Glycerol & $1: 2$ \\
DES-7 & Choline chloride & Propylene glycol & $1: 2$ \\
DES-8 & Choline chloride & Xylitol & $1: 1$ \\
DES-9 & Choline chloride & Urea & $1: 2$ \\
DES-10 & Choline chloride & Acetic acid & $1: 2$ \\
DES-11 & Choline chloride & Citric acid & $1: 2$ \\
\hline
\end{tabular}

\section{Results and Analysis}

3.1. HPLC Method Validation. The resolution of the chromatogram obtained by HPLC analysis of the mixed reference solution and Coptis chinensis extract was adequate. In addition, the number of theoretical plates was more than 3000 (Figure 1).

The mixed reference substance reserve solution was diluted 50, 25, 10, 5, 2.5, and 0 times, respectively. In addition, the peak area value was recorded by HPLC analysis. The calibration curve was drawn with peak area $(A)$ as ordinate $(y)$ and reference substance concentration $(\mathrm{mg} / \mathrm{mL})$ as abscissa $(x)$. The regression equations of palmatine and berberine were obtained as $y=33054 x-11.777(r=0.9997)$, the linear range was $0.011 \sim 0.572 \mathrm{mg} / \mathrm{mL} ; y=36898 x-352.3$ $(r=0.9996)$ and the linear range was $0.023 \sim 1.140 \mathrm{mg} / \mathrm{mL}$, respectively.

The same extract of Coptis chinensis was injected continuously for 6 times according to the above HPLC analysis conditions. Besides, the RSD values of palmatine and berberine peak area were $1.45 \%$ and $1.33 \%$, respectively, indicating that the instrumental precision was good. The RSD values of palmatine and berberine peak area calculated at 0 , $2,4,6,12,24$, and $48 \mathrm{~h}$ were $0.78 \%$ and $1.02 \%$, respectively, according to the above HPLC analysis conditions, indicating that the solution was stable within $48 \mathrm{~h}$.

Six samples of Coptis chinensis were analyzed (50 mg). In addition, $1.5 \mathrm{~mL}$ of DES-5 (water content 30\%) was added, and Coptis chinensis was extracted according to the method described in Section 2.5. The average contents of palmatine and berberine were calculated to be $14.98 \mathrm{mg} / \mathrm{g}$ and $55.04 \mathrm{mg}$ by HPLC analysis, and the RSD values were $1.77 \%$ and $1.62 \%$, respectively. This indicated that the extraction method had good repeatability.

Six samples of Rhizoma Coptidis with known content, about $25 \mathrm{mg}$ each, were precisely removed from palmatine reference solution (concentration $0.745 \mathrm{mg} / \mathrm{mL}$ ) $0.5 \mathrm{~mL}$ and berberine reference solution (concentration $0.555 \mathrm{mg} / \mathrm{mL}$ ) $2.5 \mathrm{~mL}$ in the same centrifuge tube. The well-proportioned Coptis chinensis powder was added, and DES-5 (water content $30 \%$ ) $1.5 \mathrm{~mL}$ was added after steaming and drying the solvent in water bath. Coptis chinensis extract was extracted according to the method described in Section 2.5 and the extract was analyzed by HPLC. The average recoveries of palmatine and berberine were $100.1 \%$ and $98.7 \%$, respectively, and the RSDs were $1.85 \%$ and $1.97 \%$, respectively.

3.2. Effects of Different DESs, Molar Ratio, and Moisture Content of DES on the Contents of Palmatine and Berberine. The composition of DESs determines its physical and chemical properties, which affects its extraction efficiency of natural compounds. The effects of 11 kinds of DESs (Table 1) on the contents of palmatine and berberine were studied. As shown in Figure 2(a), DES-5, the combination of choline chloride and phenol, was the most efficient in extracting palmatine and berberine from Coptis chinensis.

The viscosity of most DES decreases with the increase of temperature and the increase of hydrogen-bonded donors [11]. As can be seen from Figure 2(b), the extraction amount of palmatine and berberine from Coptis chinensis is the most when the molar ratio of choline chloride to phenol is $1: 3$. Less intramolecular hydrogen bonds may be formed, and the viscosity is very high when the ratio of choline chloride is low. It is not conducive to full contact with Coptis chinensis powder. The viscosity decreases and the extraction amount increases with the increase of phenol. The extraction amount of palmatine and berberine decreases when the molar ratio is more than $1: 4$. It may be caused by the instability of eutectic solvent and the decrease of hydrogen bond. Therefore, the molar ratio of $1: 3$ resulted more beneficial to the extraction of effective components of Coptis chinensis.

The addition of water reduces the viscosity of DESs. Moreover, the decrease of viscosity is beneficial to improve the cavitation effect of ultrasonic wave and reduce the loss of ultrasonic wave in the process of propagation [22]. Consequently, it is conducive to the extraction of berberine and palmatine in Coptis chinensis in a certain range. However, if we continue to increase the water content; it may affect the intermolecular hydrogen bond of DESs, change the structure of DESs, and reduce the solubility of effective components of Coptis chinensis. In this study, the effects of choline chloridephenol eutectic solvents with different amounts of water on the extraction of palmatine and berberine from Coptis chinensis were investigated. As shown in Figure 2(c), the extraction amount of palmatine and berberine in Coptis chinensis also increased gradually with the increase of water 


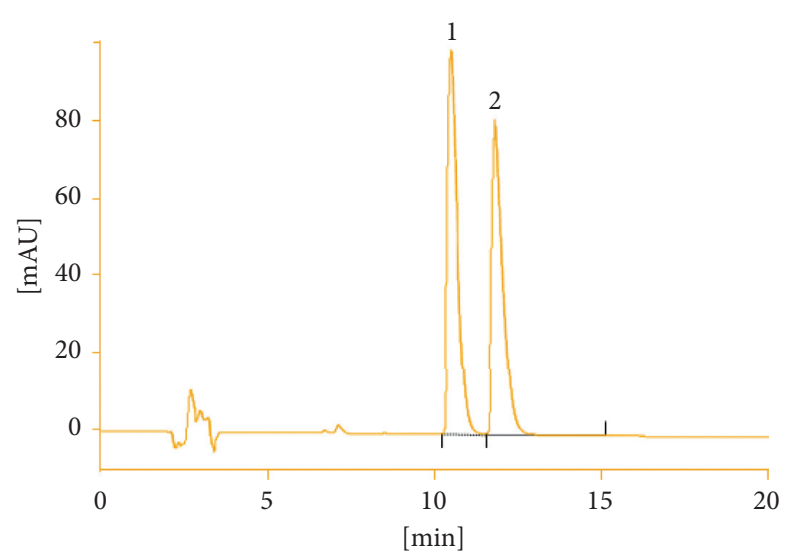

(a)

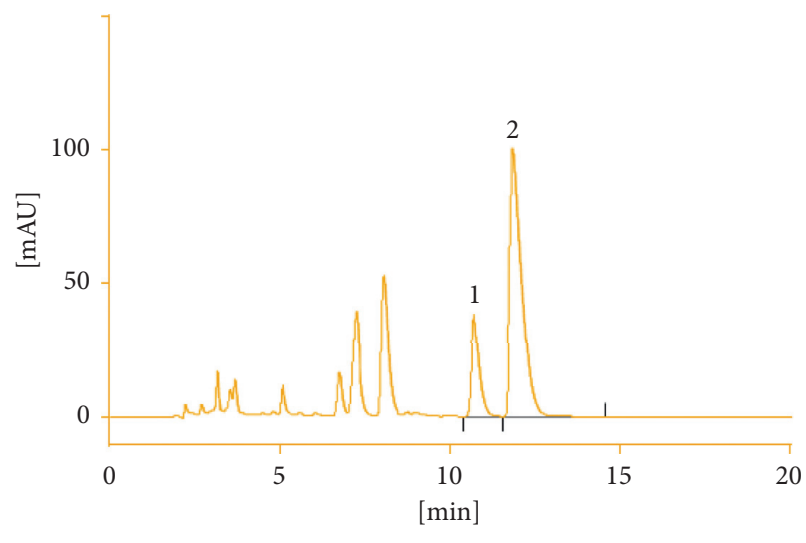

(b)

FIGURE 1: HPLC chromatogram (1, palmatine; 2, berberine).

content. However, it changed when the water content was more than $40 \%$, and the extraction efficiency of Coptis chinensis decreased. This indicated that the water content of DES has a great influence on the extraction efficiency of Coptis chinensis.

3.3. Effect of Material-Liquid Ratio. The amount of solvent determines the efficiency to a certain extent of the whole extraction process. It can be seen from Figure 2(d) that the content of palmatine and berberine increases with the increase of material-liquid ratio. The extraction amount reached the highest point when the ratio of material to liquid was $1: 30 \mathrm{~g} / \mathrm{mL}$. In addition, the contents of palmatine and berberine were almost unchanged when the ratio of material to liquid continued to increase. This may be because palmatine and berberine have been basically dissolved after the ratio of material to liquid is increased to a certain extent. If we simply increase the ratio of solvent, it has no obvious effect on the extraction of palmatine and berberine. Therefore, $1: 30 \mathrm{~g} / \mathrm{mL}$ was selected as the ratio of material to liquid for the extraction of palmatine and berberine from Coptis chinensis.

\subsection{Effect of Ultrasonic Time, Ultrasonic Power, and Ultra-} sonic Temperature. Ultrasonication is an important step in the whole extraction process; sonication time directly affects the results. It can be concluded from Figure 2(e) that the extraction amount of palmatine and berberine from Coptis chinensis increases with the extension of time. The extraction efficiency of DESs reached the higher value with $30 \mathrm{~min}$ of sonication time. Despite this if time is too long, it may lead to the decomposition of active components of Coptis chinensis or other chemical reactions may occur. Consequently, the extraction value of palmatine and berberine decreases after $40 \mathrm{~min}$.

Ultrasonic power also affects the extraction. Although ultrasonication can promote the dissolution of active substances, high ultrasonic power not only increases the experimental cost but also may cause changes in the structure of active substances. In the range of $100 \mathrm{~W}$ to $400 \mathrm{~W}$, the content of palmatine and berberine in Coptis chinensis was the highest when the ultrasonic power reached $200 \mathrm{~W}$. Besides, more than $200 \mathrm{~W}$ had almost no effect on the extraction effect, so the power of ultrasonic extraction was set at 200W (Figure 2(f)).

The appropriate extraction temperature is also an important parameter affecting the extraction of DES. Too high temperature may lead to the decomposition of the extract. Besides, if the temperature is too low, the extraction is not complete. It can be concluded from Figure $2(\mathrm{~g})$ that the extraction content of palmatine and berberine in Coptis chinensis increased with the increase of temperature. In addition, it decreased when the extraction temperature exceeded $70^{\circ} \mathrm{C}$. Moreover, it can be seen that increasing the temperature to $60^{\circ} \mathrm{C}$ can reduce the surface tension and viscosity of DES and make the extract easier to filter.

3.5. Response Surface Test Results and Analysis. According to the single-factor experimental results and Box-Behnken design principles, the test conditions are selected using the software Design-Expert 8.0.6. Moreover, the influencing factors such as moisture content $(\mathrm{A} / \%)$, molar ratio (B), ultrasonic temperature $\left(\mathrm{C} /{ }^{\circ} \mathrm{C}\right)$, and sonication time $(\mathrm{D} / \mathrm{min})$ were selected as independent variables, and palmatine $(Y 1$, $\mathrm{mg} / \mathrm{g})$, berberine $(Y 2, \mathrm{mg} / \mathrm{g})$, and total content $(Y 3, \mathrm{mg} / \mathrm{g})$ were selected as evaluation indexes. The central point was tested in quintuplicate, the response surface analysis model was of 29 test sites with 4 factors and 3 levels. Response surface establishes optimum values for the control variables that will result in maximum activity or yield over the region evaluated in a specific experimental design [23]. The experimental design and response values are shown in Table 2, and the results of analysis of variance are presented in Table 3.

The quadratic polynomial equations of the response values $Y 1, Y 2$, and $Y 3$ can be obtained by Design-Expert software and regression fitting of various factors. 

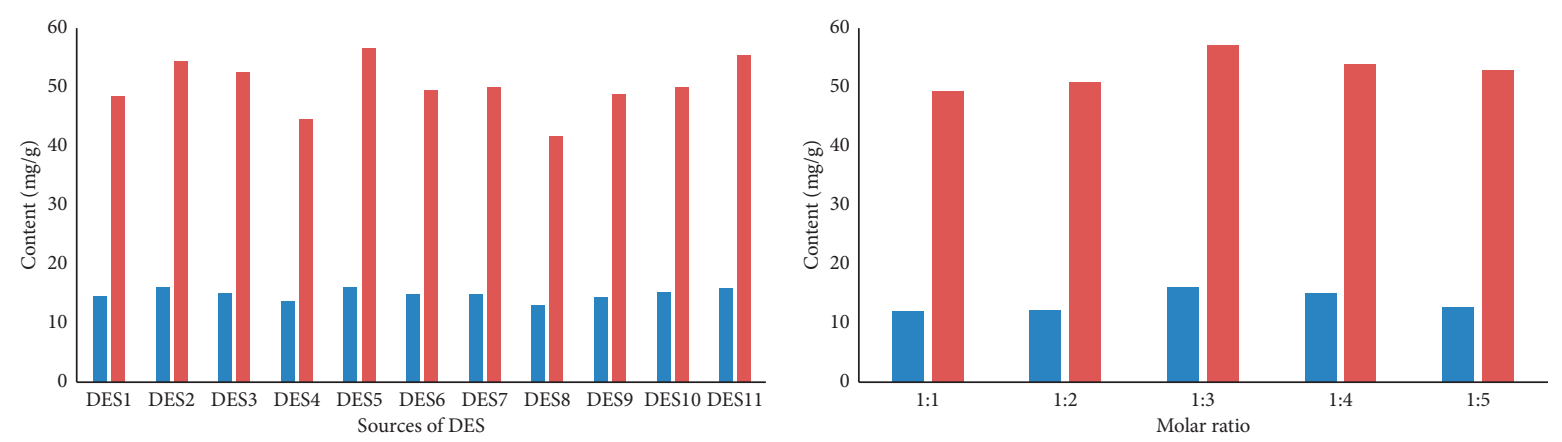

- Palmatine

- Palmatine

- Berberine

(a)
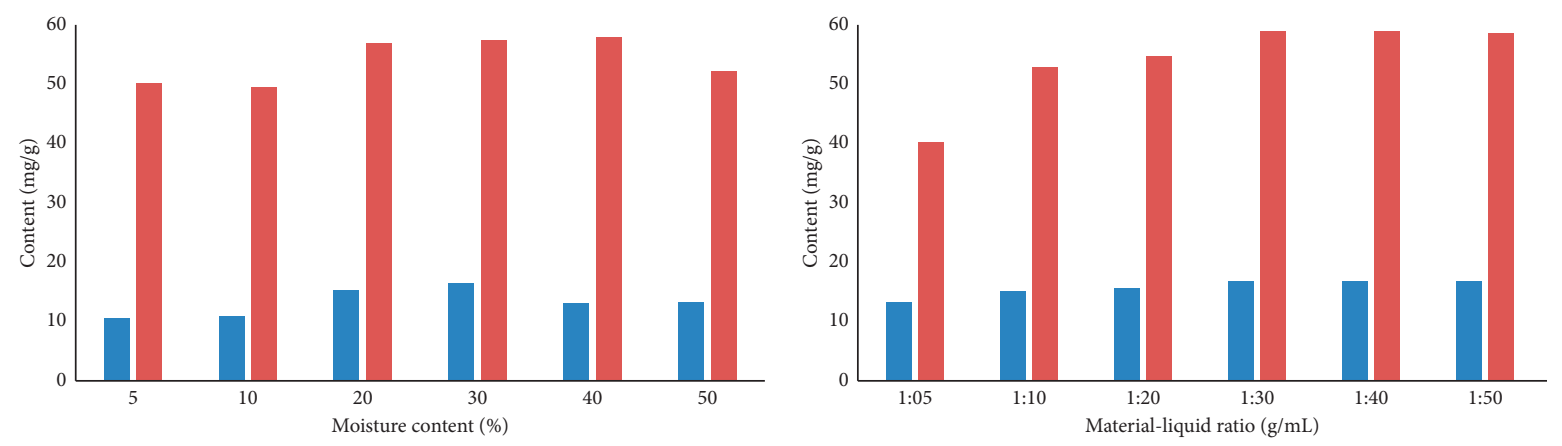

n Palmatine

- Palmatine

- Berberine

(c)

(d)
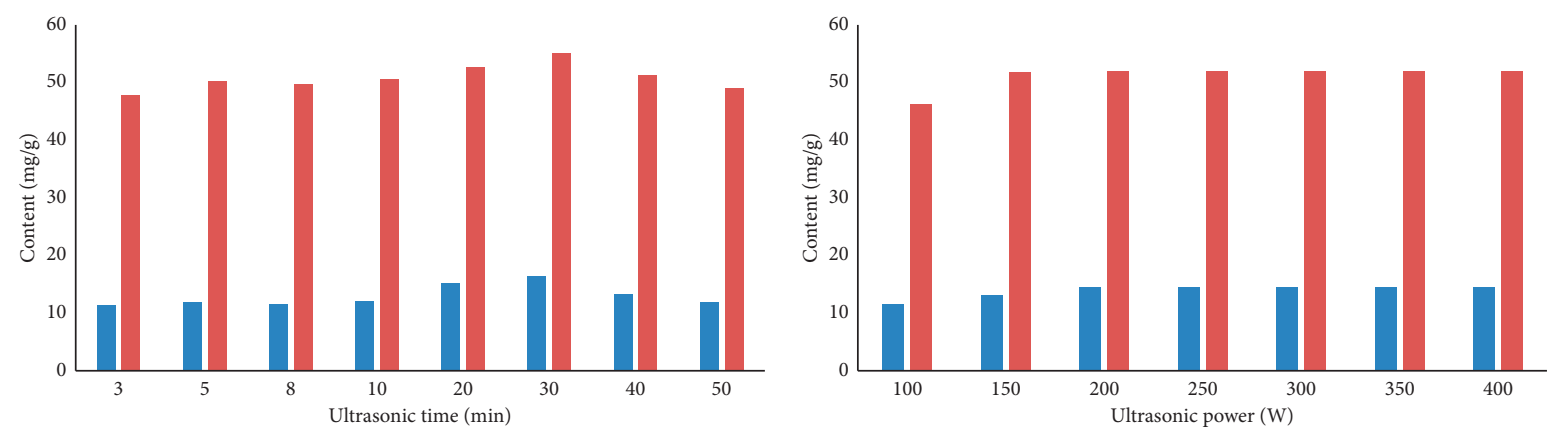

v Palmatine

- Berberine

v Palmatine

- Berberine

(e)

(f)

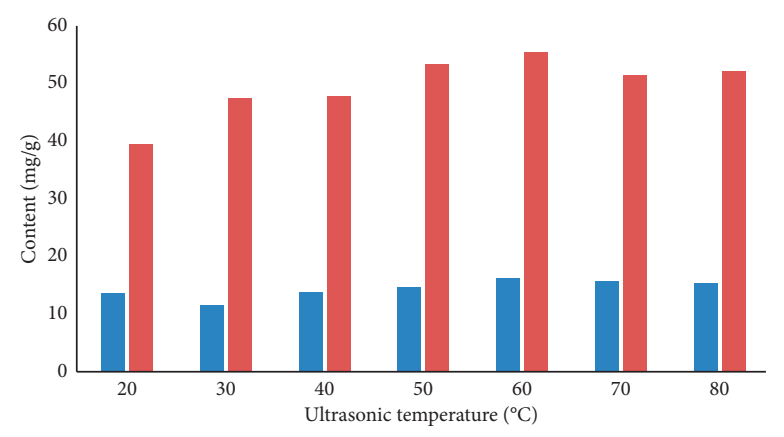

- Palmatine

- Berberine

(g)

FIGURE 2: Effects of different types of DES (a), different ratios of DES (b), different moisture contents (c), different material/liquid ratios (d), different extraction times (e), different ultrasound powers (f), and different ultrasound temperatures ( $\mathrm{g}$ ) on the extraction rates of palmatine and berberine. 
TABLE 2: Design scheme and results of response surface test.

\begin{tabular}{|c|c|c|c|c|c|c|c|}
\hline No. & Factor A & Factor B & Factor C & Factor D & Palmatine (mg/g) & Berberine (mg/g) & Total (mg/g) \\
\hline 1 & 30 & $1: 3$ & 60 & 30 & 16.3318 & 57.3551 & 73.6869 \\
\hline 2 & 40 & $1: 4$ & 60 & 30 & 11.0627 & 52.7157 & 63.7784 \\
\hline 3 & 30 & $1: 2$ & 50 & 30 & 10.6435 & 50.1267 & 60.7702 \\
\hline 4 & 30 & $1: 4$ & 70 & 30 & 10.7245 & 50.6206 & 61.3451 \\
\hline 5 & 20 & $1: 3$ & 60 & 40 & 13.7411 & 54.3042 & 68.0453 \\
\hline 6 & 40 & $1: 3$ & 70 & 30 & 13.3213 & 54.7225 & 68.0438 \\
\hline 7 & 40 & $1: 2$ & 60 & 30 & 11.1041 & 52.7099 & 63.814 \\
\hline 8 & 30 & $1: 3$ & 60 & 30 & 16.2104 & 57.9285 & 74.1389 \\
\hline 9 & 20 & $1: 3$ & 60 & 20 & 13.6131 & 54.3227 & 67.9358 \\
\hline 10 & 30 & $1: 3$ & 60 & 30 & 16.1302 & 56.3247 & 72.4549 \\
\hline 11 & 30 & $1: 4$ & 60 & 20 & 11.4241 & 49.8093 & 61.2334 \\
\hline 12 & 30 & $1: 2$ & 60 & 40 & 11.5384 & 50.2042 & 61.7426 \\
\hline 13 & 20 & $1: 3$ & 50 & 30 & 13.3432 & 54.6824 & 68.0256 \\
\hline 14 & 30 & $1: 3$ & 50 & 40 & 13.1207 & 52.3281 & 65.4488 \\
\hline 15 & 20 & $1: 2$ & 60 & 30 & 11.3957 & 52.7088 & 64.10449 \\
\hline 16 & 30 & $1: 2$ & 70 & 30 & 10.7325 & 50.6117 & 61.3442 \\
\hline 17 & 40 & $1: 3$ & 50 & 30 & 13.2233 & 54.6982 & 67.9215 \\
\hline 18 & 30 & $1: 3$ & 60 & 30 & 16.2411 & 57.8209 & 74.0620 \\
\hline 19 & 30 & $1: 3$ & 60 & 30 & 16.2903 & 57.0996 & 73.3899 \\
\hline 20 & 30 & $1: 3$ & 70 & 20 & 13.2324 & 52.1982 & 65.4306 \\
\hline 21 & 30 & $1: 2$ & 60 & 20 & 11.3214 & 49.6992 & 61.0206 \\
\hline 22 & 20 & $1: 3$ & 70 & 30 & 13.2234 & 54.7222 & 67.9456 \\
\hline 23 & 30 & $1: 4$ & 50 & 30 & 10.7732 & 50.2047 & 60.9779 \\
\hline 24 & 30 & $1: 3$ & 50 & 20 & 13.1235 & 52.172 & 65.2955 \\
\hline 25 & 30 & $1: 4$ & 60 & 40 & 11.1235 & 50.2113 & 61.3348 \\
\hline 26 & 20 & $1: 4$ & 60 & 30 & 11.2514 & 52.6925 & 63.9439 \\
\hline 27 & 40 & $1: 3$ & 60 & 20 & 13.6322 & 54.3141 & 67.9463 \\
\hline 28 & 30 & $1: 3$ & 70 & 40 & 13.1861 & 52.1978 & 65.3839 \\
\hline 29 & 40 & $1: 3$ & 60 & 40 & 13.5941 & 54.3248 & 67.9189 \\
\hline
\end{tabular}

TABLE 3: Bivariate multiple regression fitting analysis of variance results of Box-Behnken experimental design.

\begin{tabular}{|c|c|c|c|c|c|}
\hline Source & Sum of squares & $\mathrm{df}$ & Mean square & F value & $p$ value \\
\hline Model & 517.23 & 14 & 36.94 & 234.88 & $<0.0001$ \\
\hline A, moisture content & 0.03 & 1 & 0.03 & 0.18 & 0.6805 \\
\hline $\mathrm{B}$, molar ratio & 0.00 & 1 & 0.00 & 0.02 & 0.8962 \\
\hline C, ultrasonic temperature & 0.09 & 1 & 0.09 & 0.59 & 0.4558 \\
\hline $\mathrm{D}$, ultrasonic time & 0.09 & 1 & 0.09 & 0.54 & 0.4735 \\
\hline $\mathrm{AB}$ & 0.00 & 1 & 0.00 & 0.02 & 0.8770 \\
\hline $\mathrm{AC}$ & 0.01 & 1 & 0.01 & 0.07 & 0.8024 \\
\hline $\mathrm{AD}$ & 0.00 & 1 & 0.00 & 0.03 & 0.8654 \\
\hline $\mathrm{BC}$ & 0.01 & 1 & 0.01 & 0.07 & 0.7981 \\
\hline $\mathrm{BD}$ & 0.10 & 1 & 0.10 & 0.61 & 0.4470 \\
\hline $\mathrm{CD}$ & 0.01 & 1 & 0.01 & 0.06 & 0.8046 \\
\hline$A^{2}$ & 13.83 & 1 & 13.83 & 87.90 & $<0.0001$ \\
\hline $\mathrm{B}^{2}$ & 437.40 & 1 & 437.40 & 2780.84 & $<0.0001$ \\
\hline $\mathrm{C}^{2}$ & 111.51 & 1 & 111.51 & 708.98 & $<0.0001$ \\
\hline $\mathrm{D}^{2}$ & 106.18 & 1 & 106.18 & 675.03 & $<0.0001$ \\
\hline Residual & 2.20 & 14 & & & \\
\hline Lack of fit & 0.35 & 10 & 0.01 & 0.74 & 0.9995 \\
\hline Pure error & 1.85 & 4 & & & \\
\hline Cor total & 519.43 & 28 & & & \\
\hline
\end{tabular}

$Y 1$

$(\mathrm{mg} / \mathrm{g})=16.24-0.053 \mathrm{~A}-0.031 \mathrm{~B}+0.016 \mathrm{C}-0.082 \mathrm{C}$ $0.0035 \mathrm{D}+0.026 \mathrm{AB}+0.054 \mathrm{AC}-0.042 \mathrm{AD}-0.034 \mathrm{BC}-$ $0.13 \mathrm{BD}-0.011 \mathrm{CD}-1.28 \mathrm{~A}^{2}-3.71 \mathrm{~B}^{2}-1.77 \mathrm{C}^{2}-1.27 \mathrm{D}^{2}$ $\left(R^{2}=0.9982, p<0.001\right.$, lack of fit $\left.=0.1849>0.05\right)$;
$Y 2$

$(\mathrm{mg} / \mathrm{g})=57.31+0.0049 \mathrm{~A}+0.016 \mathrm{~B}+0.082 \mathrm{C}+$ $0.078 \mathrm{D}+0.004 \mathrm{AB}-0.0039 \mathrm{AC}+0.0073 \mathrm{AD}-0.017 \mathrm{BC}$ $-0.026 \mathrm{BD}-0.0083 \mathrm{CD}-0.17 \mathrm{~A}^{2}-4.50 \mathrm{~B}^{2}-2.339 \mathrm{C}^{2}-$ $2.79 \mathrm{D}^{2}\left(R^{2}=0.9889, p<0.001\right.$, lack of fit $\left.=0.9995>0.05\right)$; 

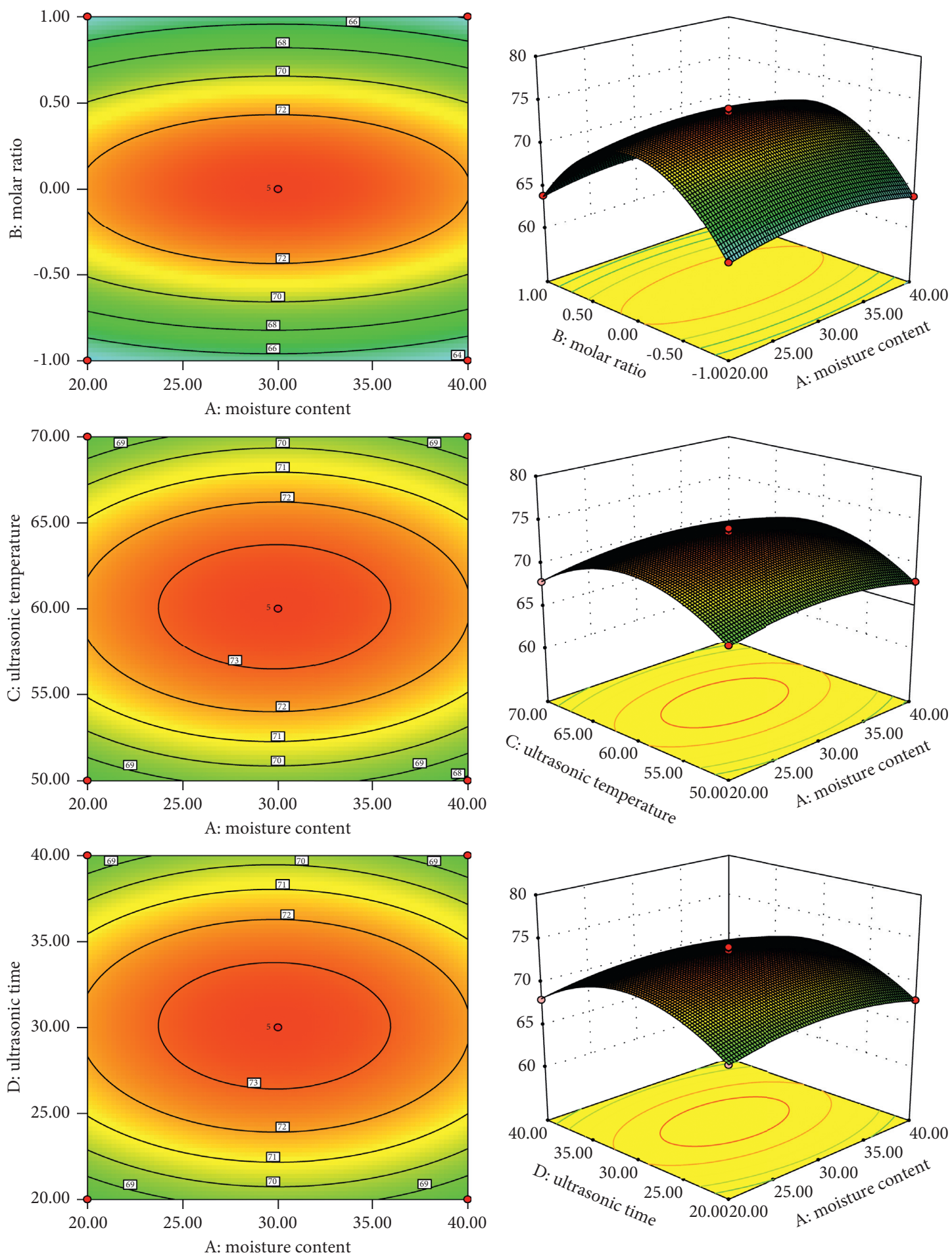

(a)

Figure 3: Continued. 

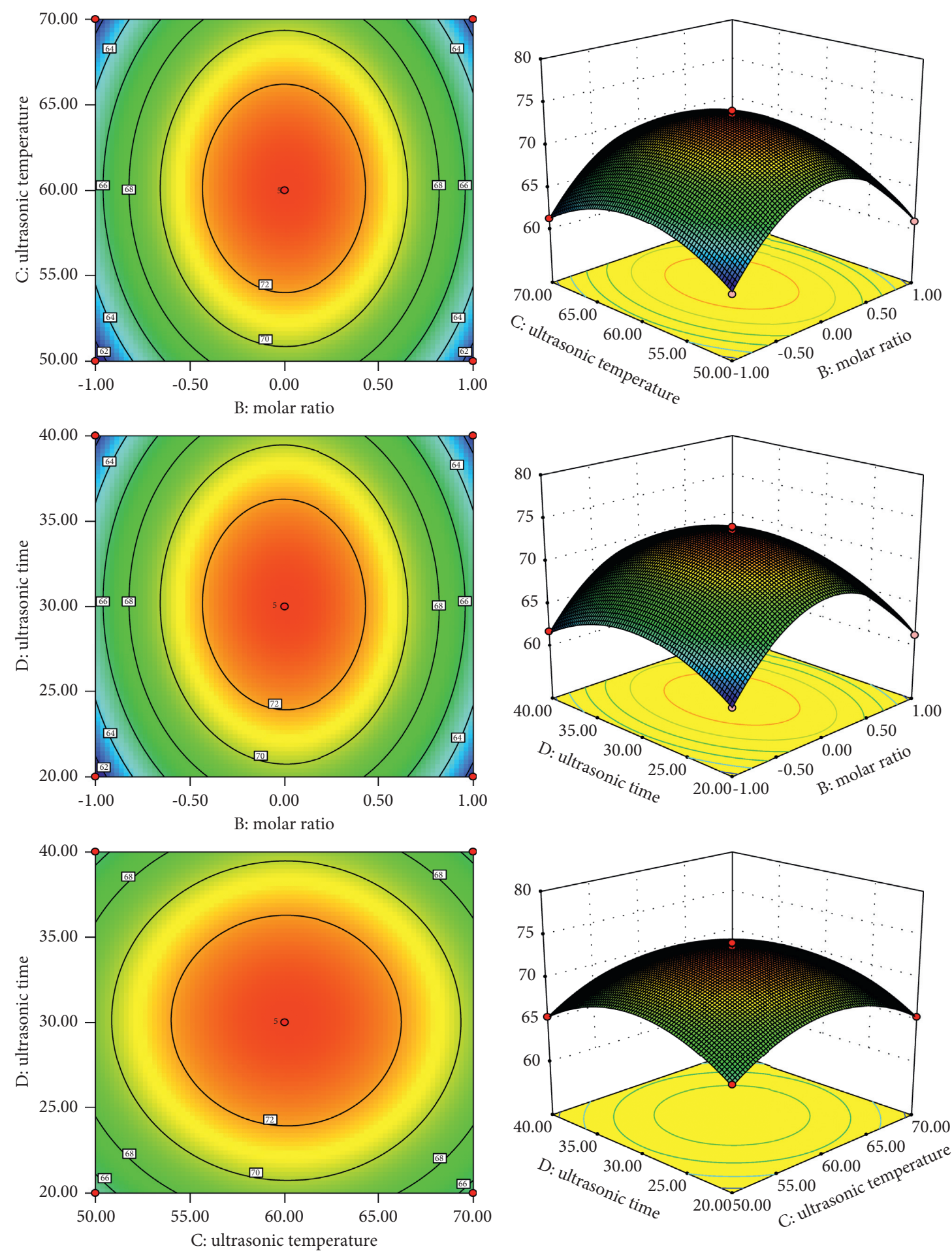

(b)

FIgURE 3: Response surface drawings of total content analyzed by interaction of two factors.

Y3

$(\mathrm{mg} / \mathrm{g})=73.55-0.048 \mathrm{~A}-0.015 \mathrm{~B}+0.088 \mathrm{C}+$ $0.084 \mathrm{D}+0.031 \mathrm{AB}+0.051 \mathrm{AC}-0.034 \mathrm{AD}-0.052 \mathrm{BC}-$ $0.16 \mathrm{BD}-0.050 \mathrm{CD}-1.46 \mathrm{~A}^{2}-8.21 \mathrm{~B}^{2}-4.15 \mathrm{C}^{2}-4.05 \mathrm{D}^{2}$ $\left(R^{2}=0.9958, p<0.001\right.$, lack of fit $\left.=0.9995>0.05\right)$.
According to the results, the three models were extremely significant $(p<0.001)$, and the misfit items were all $>0.05$, and the correlation coefficient $R^{2}>0.98$. This indicated that the fitting degree of the model was high, and the equation could better reflect the relationship between the factors and the response surface. 


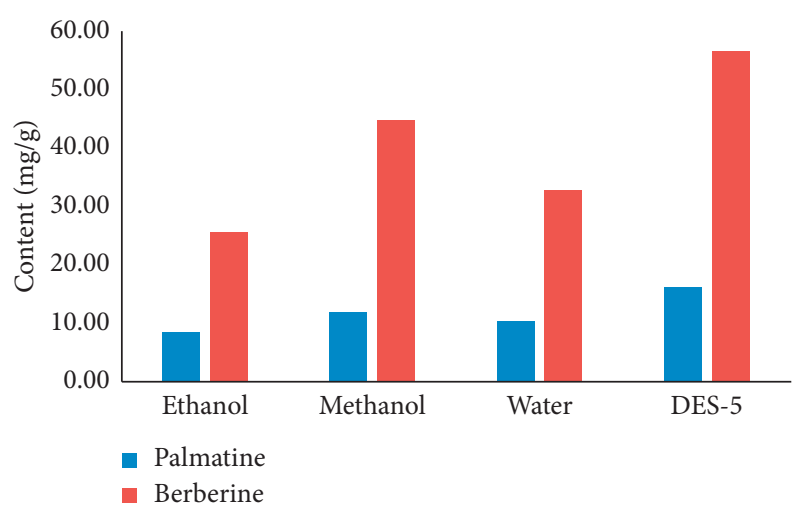

Figure 4: Comparison of different extraction methods.

We can comprehensively evaluate the $3 \mathrm{D}$ response surface map and 2D contour map of the total content of palmatine and berberine and directly view the range of the best process parameters by using Design-Expert 8.0.6 software and according to the quadratic polynomial equation (Figure 3). As can be seen from the figure, the $\mathrm{BD}$ contours were oval, and the interaction is obvious.

The best process parameters can be obtained by solving the quadratic polynomial equation, and the mathematical model prediction of palmatine and berberine content can be calculated. It was predicted that the best extraction process parameters were as follows: water content was $29.83 \%$, molar ratio was $1: 3$, temperature was $60.10^{\circ} \mathrm{C}$, and ultrasonic time was $30.09 \mathrm{~min}$. Under this condition, the theoretical values of palmatine and berberine content were $16.2412 \mathrm{mg} / \mathrm{g}$ and $57.3066 \mathrm{mg} / \mathrm{g}$, respectively. Combined with the actual needs, it was finally determined that the extraction process was as follows: water content was 30\%, molar ratio was $1: 3$, temperature was $60^{\circ} \mathrm{C}$, and ultrasonic time was $30 \mathrm{~min}$. Three samples of Coptis chinensis were selected, and the contents of palmatine and berberine were calculated according to the optimum process parameters. The results showed that the average contents of palmatine and berberine were 16.7145 and $57.4013 \mathrm{mg} / \mathrm{g}$, respectively. It was very close to the predicted value of the model. Therefore, the prediction of the model was accurate and reliable, and the best extraction process was desirable.

3.6. Comparison of Different Extraction Methods of Coptis chinensis. Water and organic solvents are used as extraction solvents for the extraction of active compounds of traditional Chinese medicine at present. In addition, the main extraction methods are solvent extraction [24], ultrasonic extraction [25], and macroporous resin adsorption, among others [26]. Herein, an extraction process of ultrasoundassisted eutectic solvent was optimized. In addition, the results were compared with the extraction results of common extraction solvents such as water, methanol, and ethanol. It can be seen from the experimental process that this method has mild process conditions and does not need high temperature, ensures the stability of the extracted components, has a short extraction time, improves extraction efficiency, and saves time and cost $[27,28]$. As can be seen in Figure 4, the extraction effect of the optimized ultrasound-assisted eutectic solvent extraction method was slightly better than those that use conventional extraction solvents. In addition, the low eutectic solvent is a green environmental protection natural solvent $[29,30]$.

\section{Conclusion}

In this study, Coptis chinensis was used as the research object, and low eutectic solvent system was constructed by using low eutectic solvent as the extractant, choline chloride as the hydrogen bond acceptor, and phenol as the hydrogen bond donor. The molar ratio of two eutectic solvents was 1 : 3 , the water content of eutectic solvent system was $30 \%(\mathrm{v} /$ $\mathrm{v}$ ), and the ratio of material to liquid was $1: 30 \mathrm{~g} / \mathrm{mL}$. Moreover, the extraction rate of berberine and palmatine was the highest with a sonication time of $30 \mathrm{~min}$. The extraction rate of berberine and palmatine was affected by the ratio of material to liquid, temperature, and time in ultrasonic-assisted eutectic solvent extraction. DESs possess many preferable characteristics, including safety, nontoxicity, biodegradability, sustainability, low cost, and easy preparation, and it avoids the harm to the extraction personnel and environmental pollution.

The extract can be used in food, medicine, and other fields. It reflects the advantages of high yield and high speed of extracting active compounds by low eutectic solvent. This study not only provides a new method for the extraction of alkaloids from Coptis chinensis but also provides a reference for the extraction of active components of traditional Chinese medicine.

\section{Data Availability}

The main table and figure data used to support the findings of this study are included within the article.

\section{Conflicts of Interest}

The authors declare that they have no conflicts of interest.

\section{Acknowledgments}

This work was supported by a grant from "13th Five-Year Plan" Science and Technology Research Projects of Education Department of Jilin Province (No. JJKH20200904KJ), Study on quality markers of Sangbaipi Decoction based on spectrum effect relationship.

\section{References}

[1] National Pharmacopoeia Commission, Pharmacopoeia of the People's Republic of China, China Medical Science and Technology Press, Beijing, China, (in Chinese), 2015.

[2] F. Cao, X. Z. Cha, N. Xu et al., "Research progress of coptis and its drug pairs," Renowned Doctor, no. 9, pp. 330-331, 2020, (in Chinese).

[3] M. X. Zhang, X. Z. Zhang, X. Q. Zeng et al., "The history of rhizoma coptidis and its clinical application in cardio-cerebrovascular diseases," Journal of Liaoning College of Traditional Chinese Medicine, pp. 1-12, (in Chinese). 
[4] C. M. Li, C. L Zhang, L. Zhou et al., "Optimization of compound planting mode of Coptis Chinensis and different young forests at mountain areas of the southwest of Hubei," Non-wood Forest Research, vol. 31, no. 1, pp. 19-123, 2013, (in Chinese).

[5] Q. G. G. Gu, The Book of God's Agricultural Herbs, Harbin Press, Harbin, China, (in Chinese), 2007.

[6] L. Fu, Q. Fu, W. Li, and X. Yu, "Advances in the study of the chemical composition and pharmacological effects of Coptis Chinensis," Acta Chinese Medicine and Pharmacology, vol. 49, no. 2, pp. 87-92, 2021, (in Chinese).

[7] Z. P. Geng, H. J. Zheng, Y. Zhang et al., "Simultaneous determination of six alkaloids in Coptis Chinensis of different regions by RP-HPLC," Chinese Medical Journal, vol. 35, no. 19, 2010.

[8] N. Y. Yang, Q. C. Zhang, H. X. Zhu et al., "Research progress and utilization strategy on alkaloid resource chemistry of Coptidis Rhizoma," Chinese Traditional and Herbal Drugs, vol. 50, no. 20, pp. 5080-5087, 2019, (in Chinese).

[9] A. P. Abbott, G. Capper, D. L. Davies, R. K. Rasheed, and V. Tambyrajah, "Novel solvent properties of choline chloride/ urea mixturesElectronic supplementary information (ESI) available: spectroscopic data," Chemical Communications, vol. 9, no. 1, pp. 70-71, 2003

[10] Z. Y. Fang and L. M. Xing, "Eutectic solvent technology and its application," Shandong Yuean Chemical Industry, vol. 49, no. 24, pp. 136-138, 2020, (in Chinese).

[11] S. Y. Yue, X. Jin, X. Y. Dong, and C. Q. Qu, "Extraction rate of total flavonoids from Toona sinensis seeds with different deep eutectic solvents," Journal of Fujian Normal University (Philosophy and Social Sciences Edition), vol. 37, no. 2, pp. 67-71, 2020, (In Chinese).

[12] Y. C. Hou, C. F. Yao, and W. Z. Wu, "Deep eutectic solvents: green solvents for separation applications," Acta Phys-Chim Sin, vol. 34, no. 8, pp. 873-885, 2018.

[13] X. Li and K. H. Row, "Development of deep eutectic solvents applied in extraction and separation," Journal of Separation Science, vol. 39, no. 18, pp. 3505-3520, 2016.

[14] E. L. Smith, A. P. Abbott, and K. S. Ryder, "Deep eutectic solvents (DESs) and their applications," Chemical Reviews, vol. 114, no. 21, pp. 11060-11082, 2014.

[15] J. J. Zhu, Z. M. Wang, X. Y. Ma et al., "A quantitative method for simultaneous determination of four anthraquinones with one marker in Rhei Radix et Rhizoma," Chinese Herbal Medicines, vol. 4, no. 2, pp. 157-163, 2012.

[16] G. Garcia, S. Aparicio, R. Ullah et al., "Deep eutectic solvents: physicochemical properties and gas separation applications," Energy and Fuels, vol. 29, no. 4, pp. 2616-2644, 2015.

[17] J. M. Liu, H. Wan, H. Zhang et al., "Research progress on extraction of active ingredients and pretreatment of food analysis by ultrasound-assisted deep eutectic solvent method," Science and Technology of Food Industry, pp. 1-12, (In Chinese).

[18] Y. Sun, X. B. Liu, Z. W. Su et al., "Extraction of flavonoids from chickpeas by microwave-assisted eutectic solvent and its antioxidant activity," Science and Technology of Food Industry, vol. 41, no. 14, pp. 120-128, 2020.

[19] L. Alavi, S. Seidi, A. Jabbari et al., "Deep eutectic liquid organic salt as a new solvent for carrier-mediated hollow fiber liquid phase microextraction of lead from whole blood followed by electrothermal atomic absorption spectrometry," New Journal of Chemistry, vol. 41, pp. 7038-7044, 2017.

[20] S. C. Cunha and J. O. Fernandes, "Multiple mycotoxin analysis in nut products: occurrence and risk characterization," Trends in Analytical Chemistry (Reference Ed.), vol. 105, pp. 225-239, 2018.

[21] C. B. Marina, N. Ćurko, M. Tomašević, K. Kovačević Ganić, and I. Radojčić Redovniković, "Green extraction of grape skin phenolics by using deep eutectic solvents," Food Chemistry, vol. 200, pp. 159-166, 2016.

[22] O. S. Hammond, D. T. Bowron, and K. J. Edler, "The effect of water upon deep eutectic solvent nanostructure: an unusual transition from ionic mixture to aqueous solution," Angewandte Chemie International Edition, vol. 56, no. 33, 2017.

[23] H. O. Sergio et al., "Whole-cell bioconversion of citrus flavonoids to enhance their biological properties," Studies in Natural Products Chemistry, vol. 61, pp. 335-367, 2019.

[24] H. H. Yu and D. Z. Zhou, "Chinese herbal medicine extraction method-solvent extraction method," Modern Animal Husbandry Science \& Technology, no. 8, p. 203, 2012 (in Chinese).

[25] C. Y. Dong and J. Chen, "Research progress on ultrasonic extraction of effective ingredients from botanicals," Journal of Traditional Chinese Medicine, vol. 39, no. 7, pp. 1473-1477, 2017, (in Chinese).

[26] H. K. Zhang, "Purification of polysaccharides extract from poria cocos using macroporous resin," Food Research and Development, vol. 38, no. 23, pp. 67-71, 2017, (in Chinese).

[27] C. Jun, C. Jiarui, W. Huimin et al., "Solubility improvement of phytochemicals using (natural) deep eutectic solvents and their bioactivity evaluation - ScienceDirect," Journal of Molecular Liquids, vol. 318, p. 318, 2020.

[28] M. Yang, "Efficient extraction of bioactive flavonoids from ginkgo biloba leaves using deep eutectic solvent/water mixture as green media," Chemical and Biochemical Engineering Quarterly, vol. 32, no. 3, pp. 315-324, 2018.

[29] J. Cao, L. Chen, M. Li et al., "Efficient extraction of proanthocyanidin from Ginkgo biloba leaves employing rationally designed deep eutectic solvent-water mixture and evaluation of the antioxidant activity," Journal of Pharmaceutical and Biomedical Analysis, vol. 158, no. 5, pp. 317-326, 2018.

[30] J. Cao, L. Chen, M. Li et al., "Two-phase systems developed with hydrophilic and hydrophobic deep eutectic solvents for simultaneously extracting various bioactive compounds with different polarities," Green Chemistry, vol. 10, p. 1039, 2018. 